\title{
Leading Lyapunov Vectors of a Turbulent Baroclinic Jet in a Quasigeostrophic Model
}

\author{
CHRIS SNYDER \\ National Center for Atmospheric Research,* Boulder, Colorado \\ Thomas M. HAMILL \\ NOAA-CIRES Climate Diagnostics Center, Boulder, Colorado
}

(Manuscript received 7 December 2001, in final form 30 August 2002)

\section{ABSTRACT}

\begin{abstract}
Leading Lyapunov exponents and vectors are calculated for a turbulent baroclinic jet in a quasigeostrophic model with $O\left(10^{5}\right)$ degrees of freedom. The leading exponent is close to 0.4 day $^{-1}$, and the unstable subspace has dimension between 30 and 40. The leading Lyapunov vectors exhibit a strong correlation of their potential vorticity (PV) with the PV gradients of the unperturbed flow. These perturbations do not, however, appear to be instabilities of smaller scale on the turbulent flow. Instead, they share the scales of the flow itself (at least if measured along PV contours) and often simply represent local phase shifts or displacements of existing features in the flow. Singular vectors constrained to the subspace of Lyapunov vectors are also calculated. Maximum amplification factors over 2 days are, on average, about 6, 7.5, and 9 (compared to the factor of 2 implied by the leading exponent) for subspaces of the leading 20,35, and 60 Lyapunov vectors, respectively.
\end{abstract}

\section{Introduction}

Numerical models typically represent the governing equations for the atmosphere or ocean as a set of coupled, nonlinear ordinary differential equations. Given a (time dependent) solution from a certain initial condition, a natural question is then the stability of that solution to small perturbations of the initial condition. In this problem, which generalizes familiar linear stability analyses for steady flows, the Lyapunov vectors (LVs) and Lyapunov exponents are the counterparts of the linear modes and their growth rates; precise definitions will be given below. This article presents the leading LVs and exponents for a damped and driven quasigeostrophic flow with broad similarity to the atmospheric midlatitude jet. Special emphasis will be given to the structure of the LVs and their relation to the original, unperturbed flow.

Aside from their theoretical interest, the Lyapunov exponents provide information on the rate at which forecast errors grow, and the LVs on the form that those errors will assume, at least in the case that the forecast model is reasonably accurate and the forecast errors are

* The National Center for Atmospheric Research is sponsored by the National Science Foundation.

Corresponding author address: C. Snyder, NCAR, P.O. Box 3000, Boulder, CO 80307-3000.

E-mail: chriss@ucar.edu not too large. The LVs and exponents are therefore also of interest in areas such as ensemble forecasting (Toth and Kalnay 1997) and data assimilation (Swanson et al. 1998). Our specific motivation for calculating the leading LVs was as an aid to understanding the evolution of ensembles of random initial perturbations (Snyder et al. 2003) and the statistical characteristics of analysis error (Hamill et al. 2002) in this same quasigeostrophic model.

Excluding results from low-order models, little is known about the Lyapunov stability of atmospheric flows. Several studies have employed the same threelayer, quasigeostrophic, hemispheric model truncated at T21 (Vannitsem and Nicolis 1997; Swanson et al. 1998; Reynolds and Errico 1999). This note complements and extends those previous studies by characterizing the structure of the leading Lyapunov vectors and their relation to the unperturbed flow, and by using a different quasigeostrophic model, with different geometry, different forcing and more degrees of freedom.

\section{Background}

To fix ideas, let the unperturbed flow evolve according to

$$
d \overline{\mathbf{x}} / d t=\mathbf{f}(\overline{\mathbf{x}}),
$$

where $\overline{\mathbf{x}}(t)$ is the state of the flow in some discrete, finitedimensional representation (such as spectral components or gridpoint values). We will comment in the con- 
cluding section on the relation of results from finitedimensional systems to infinite-dimensional or continuous systems such as the atmosphere.

Infinitesimally small perturbations $\mathbf{x}$ then satisfy $d \mathbf{x} /$ $d t=\mathbf{A x}$ with $\mathbf{A}$ given by the Jacobian matrix of $\mathbf{f}$ with respect to $\overline{\mathbf{x}}$; this relation may in turn be integrated over a time interval $[\tau, t]$ to yield

$$
\mathbf{x}(t)=\mathbf{M}(\overline{\mathbf{x}}, \tau, t) \mathbf{x}(\tau)
$$

where $\mathbf{M}$ is the propagator or resolvent matrix and depends on the unperturbed solution and the time interval.

We are then concerned with the behavior of solutions of (2) as $t-\tau \rightarrow \infty$. An excellent introduction to the theory as well as further references can be found in Legras and Vautard (1995). Here, we briefly review the points of importance to what will follow.

There are two key results. First, almost any initial perturbation will amplify exponentially at a rate that tends, when averaged over a sufficiently long interval, to a specific values $\lambda_{1}$, which is called the leading Lyapunov exponent. This exponent is a property only of (1) and is independent of both the norm chosen to measure the perturbation amplitude (for finite-dimensional systems) and the specific unperturbed solution $\overline{\mathbf{x}}$ (except for a set of measure zero). Second, almost any perturbation initialized far in the past will converge at a future time $t$ to a specific direction, given by the unit vector $\mathbf{w}_{1}(t)$. This is the leading LV, which depends on $t$ and implicitly on $\overline{\mathbf{x}}$.

More generally, there exists a sequence of exponents $\left\{\lambda_{i}\right\}$ and a corresponding set of orthonormal vectors $\left\{\mathbf{w}_{i}\right\}$ such that, given (almost) any set of $N$ initial perturbations, the hypervolume defined by these perturbations grows, on average, exponentially at the rate $\sum_{i=1}^{N} \lambda_{i}$, and the subspace spanned by these perturbations converges after sufficient time to span $\left\{\mathbf{w}_{1}, \ldots, \mathbf{w}_{N}\right\}$. Because we require the LVs to be orthogonal, each $\mathbf{w}_{i}$ except the first depends on the choice of inner product.

Our numerical calculation of the exponents and the corresponding LVs $\left\{\mathbf{w}_{i}\right\}$ follows the standard method (see Legras and Vautard 1995). Specifically, $N$ initial perturbations are integrated forward with the tangent linear model linearized about the unperturbed solution, with periodic orthonormalization, using a modified Gram-Schmidt procedure and the total energy inner product (as defined in Snyder et al. 2003). As the integration continues, the portion of the $i$ th perturbation that is orthogonal to the subspace of the first $i-1$ perturbations converges to $\mathbf{w}_{i}$ and its average amplification converges to $\lambda_{i}$.

\section{The unperturbed solution and the quasigeostrophic model}

The unperturbed solution consists of an interval of 480 days taken from the statistically steady state of a damped and driven quasigeostrophic model. The characteristics of the solution as well as the quasigeostrophic equations and their numerical integration are described more fully in Snyder et al. (2003).

In this model, the flow is periodic in $x$ and is confined between rigid surfaces at $y=0, y_{L}$ and $z=0, H$. The flow is driven by relaxation of the potential vorticity ${ }^{1}$ (PV) to a baroclinic zonal jet and is damped by a combination of Ekman pumping at the surface and a fourthorder numerical dissipation applied to the potential vorticity. Parameters are chosen as in Snyder et al. (2003); in particular, the zonal periodicity is $16 \times 10^{3} \mathrm{~km}$, the width $y_{L}$ of the channel is $8 \times 10^{3} \mathrm{~km}$, its depth $H$ is $10 \mathrm{~km}$, the relaxation has a timescale of 20 days, and the "relaxed" zonal jet has a maximum velocity of 60 $\mathrm{m} \mathrm{s}^{-1}$. The numerical model uses a gridpoint discretization with 128 points in $x, 64$ in $y$, and 8 in $z$.

In the statistically steady state, solutions to this model are characterized by a strong baroclinic jet in the central portion of the channel that intensifies from the surface to the lid. The flow is turbulent and exhibits an approximate power-law dependence of the velocity variance at channel center on zonal wavenumber (see Fig. 3 of the following section). Meanders or waves move along the jet from east to west, with zonal wavenumbers 3 or 4 (in units of periods per channel length) dominating. These waves organize into packets and frequently break to form cutoff eddies in both PV and streamfunction. Further discussion, together with snapshots of the flow, can be found in Snyder et al. (2003; see also Fig. 4 here).

The structure of the PV in the statistically steady state is qualitatively similar to that of the midlatitude troposphere, as discussed, for example, in Hoskins et al. (1985). In particular, the horizontal PV gradient at $z=$ $H$ is concentrated along the jet, so that the jet marks an abrupt change between regions of relatively uniform PV; the same is true to a lesser extent at the surface. Compared to those near the boundaries, however, PV gradients in the interior of the flow are small. This structure of the PV will turn out to control the structure of the leading LVs, as will be discussed in the following section.

\section{Lyapunov exponents and vectors}

\section{a. Lyapunov exponents}

Figure 1 shows estimates of the first 60 Lyapunov exponents, $\left\{\lambda_{i}, i=1, \ldots, 60\right\}$. The leading exponent implies an (asymptotic) doubling time for perturbations of about 2 days. Of the remaining exponents, 35 are positive, so that the unstable subspace has dimension approximately 36 , compared to the $O\left(10^{5}\right)$ degrees of freedom in the numerical model.

The exponents shown in Fig. 1 should be considered

\footnotetext{
${ }^{1}$ Throughout this article, PV will refer to the generalized PV that includes contributions from the potential temperature at $z=0, H$, in the manner of Bretherton (1966).
} 


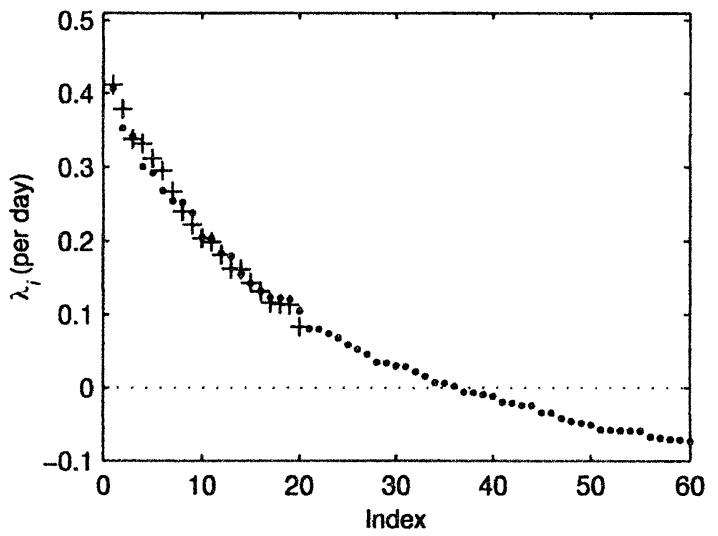

FIG. 1. The first 60 Lyapunov exponents calculated over the time interval $0 \leq t \leq 240$ days (dots), and the first 20 exponents over the interval 240 days $\leq t \leq 480$ days (crosses).

estimates only, because they are based on an integration over the finite time interval $0 \leq t \leq 240$ days. As shown in Fig. 1 (crosses), the leading exponent calculated on the interval 240 days $\leq t \leq 480$ days differs by $1 \%$ between the intervals, while the second exponent differs by $8 \%$.

Our results may be compared with those of Vannitsem and Nicolis (1997), who found a leading exponent roughly half as large $\left(0.23\right.$ day $\left.^{-1}\right)$ and an unstable subspace of more than twice the dimension (103) in a different quasigeostrophic model with $O\left(10^{3}\right)$ degrees of freedom. There are a number of possible reasons for these differences. First, our model and theirs differ in the spatial extent of the domain and also in the intensity of the forcing that maintains the flow (although a direct comparison of the intensity is problematic, as the two models are forced in markedly different ways); both factors are known to influence the Lyapunov exponents (see Cross and Hohenberg 1993, section VII.C). In addition, results from both the models are sensitive to the magnitude of dissipation. Vannitsem and Nicolis show that the leading exponent and the dimension of the unstable subspace can both be more than halved if they choose larger dissipation. In our model, halving the resolution and increasing the fourth-order diffusion (required for numerical stability) also roughly halves the exponent. We emphasize, however, that our main concern is the structure of the LVs, which we believe has generic features, and not the precise magnitude of the leading exponent or dimension of the unstable subspace, which are clearly dependent on details of the model.

\section{b. Time-averaged characteristics of Lyapunov vectors}

The variation with height of the LV amplitude is shown in Fig. 2, in terms of both potential enstrophy (that is, the squared PV) and total energy. Values shown are averages over the first $20 \mathrm{LVs}$ and over 21 times, each separated by 10 days and beginning at $t=40$ days. (Since there is little variation and no systematic trend
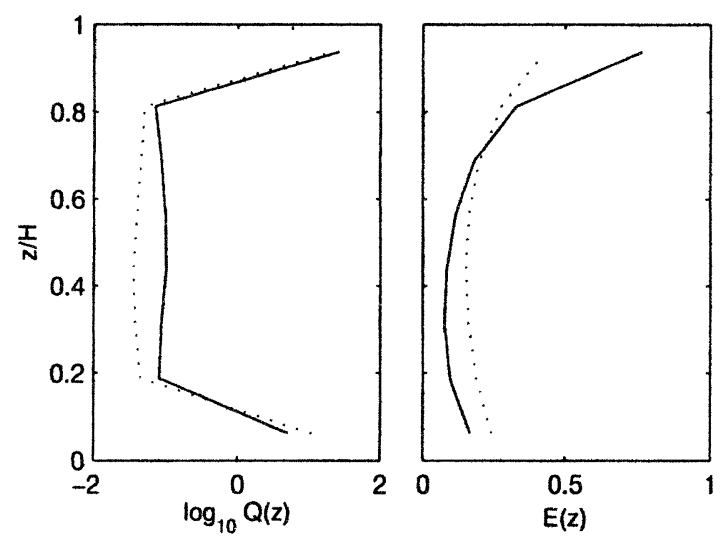

FIG. 2. (a) Time- and area-averaged potential enstrophy $Q$ as a function of height, averaged over the first 20 Lyapunov vectors (solid line) and for deviations of the unperturbed solution from its zonal mean (dotted). (b) As in (a), but for the time- and area-averaged total energy $E$. The amplitude of the Lyapunov vectors has been scaled so that the vertical integral of $Q(z)$ or $E(z)$ is equal to that based on the deviations from the zonal mean.

among the LVs, averages over the first 20 LVs suffice to characterize the structure of each.) The figure also displays potential enstrophy and energy for deviations of the unperturbed solution from its zonal mean.

The potential enstrophy of the leading LVs is dominated by the top and bottom boundaries, where rms variations of the PV are a factor of 10 larger than in the interior. Consistent with the streamfunction that would be obtained by inverting such a distribution of $\mathrm{PV}$, the energy is also maximized at top and bottom and decays into the interior. The unperturbed solution shares both these characteristics.

Thus, both the leading LVs and the unperturbed solution appear to be controlled by the PV at the boundaries with only a secondary contribution from the interior. Direct inversions of either the boundary or interior PV alone confirm that the flow arises mainly from the boundary PV, although the flow associated with the interior PV is not negligible. In this respect, both the leading LVs and the unperturbed solution resemble the midlatitude troposphere, where PV anomalies at the surface and tropopause are the primary dynamical agents (e.g., Hoskins et al. 1985; Davis 1992).

In the horizontal, the leading LVs possess noticeably finer scales than the unperturbed solution. As shown in Fig. 3, the kinetic energy spectrum at $z / H=1$ and along the channel center peaks at wavenumber 4 for the LVs instead of wavenumber 3 , and has a much shallower slope in the inertial range (roughly $5 \leq k \leq 30$ ). This shallower slope is in accord with the predictions of closures for two-dimensional turbulence; Métais and Lesieur (1986) note that perturbations should develop a $k^{-1}$ dependence in contrast to the $k^{-3}$ decay of the turbulent flow. 


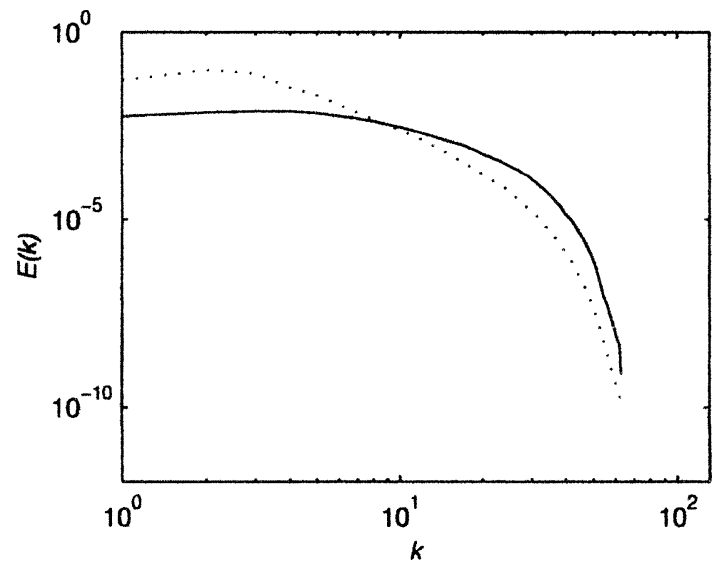

FIG. 3. The time-averaged power spectra for (nondimensional) kinetic energy at $y=y_{L} / 2$ and $z / H=1$. The solid line shows the mean of the first 20 Lyapunov vectors and the dotted shows the unperturbed solution. The amplitude of the Lyapunov vectors has been scaled so that the total energy is equal to that of the unperturbed solution.

\section{c. Instantaneous structure of Lyapunov vectors}

We next consider the relation of the first LV to the instantaneous flow in the unperturbed solution. Figure 4 shows the PV at the model top for $\mathbf{w}_{1}$ and the unperturbed solution at $t=120,140$, and 160 days; these times were chosen merely as the center of the interval over which exponents and vectors were calculated.

A striking property at each of the times is the concentration of the perturbation where the unperturbed PV gradient is large. An objective measure of the relation of $\mathbf{w}_{1}$ to unperturbed PV gradients is the correlation of the absolute value of the perturbation PV with the magnitude of the unperturbed PV gradient. This correlation averages about 0.6 over the 240-day interval; a 40-day time series is shown in Fig. 5. The concentration of $\mathbf{w}_{1}$ along the unperturbed PV gradient varies in time, but is clearly not limited to the times shown in Fig. 4.

Moreover, the perturbations are not instabilities or waves of finer scale superimposed on the unperturbed gradient: if we follow a contour of unperturbed PV, the perturbations have scales comparable or identical to the unperturbed solution. In directions parallel to the local PV gradient, however, perturbations are highly compressed. (It is this compression gives rise to the relatively flat energy spectrum of the perturbations.) Thus, $\mathbf{w}_{1}$ often simply represents a displacement or phase shift of features in the unperturbed solution.

In Fig. 4a, for example, $\mathbf{w}_{1}$ is strongly correlated with the $x$ derivative of the unperturbed solution. Adding the perturbation to the unperturbed solution would thus shift zonally both the cutoff feature at $x=8000 \mathrm{~km}$ and, because it includes an intrusion of low PV that gives rise to a positive-negative-positive pattern of the derivative, the leading edge of the trough at $x=6000 \mathrm{~km}$. Although the other examples shown in Fig. 4 are less clear-cut, the general principle is clear. Snyder and Joly (1998) and Snyder (1999) give further examples of per-
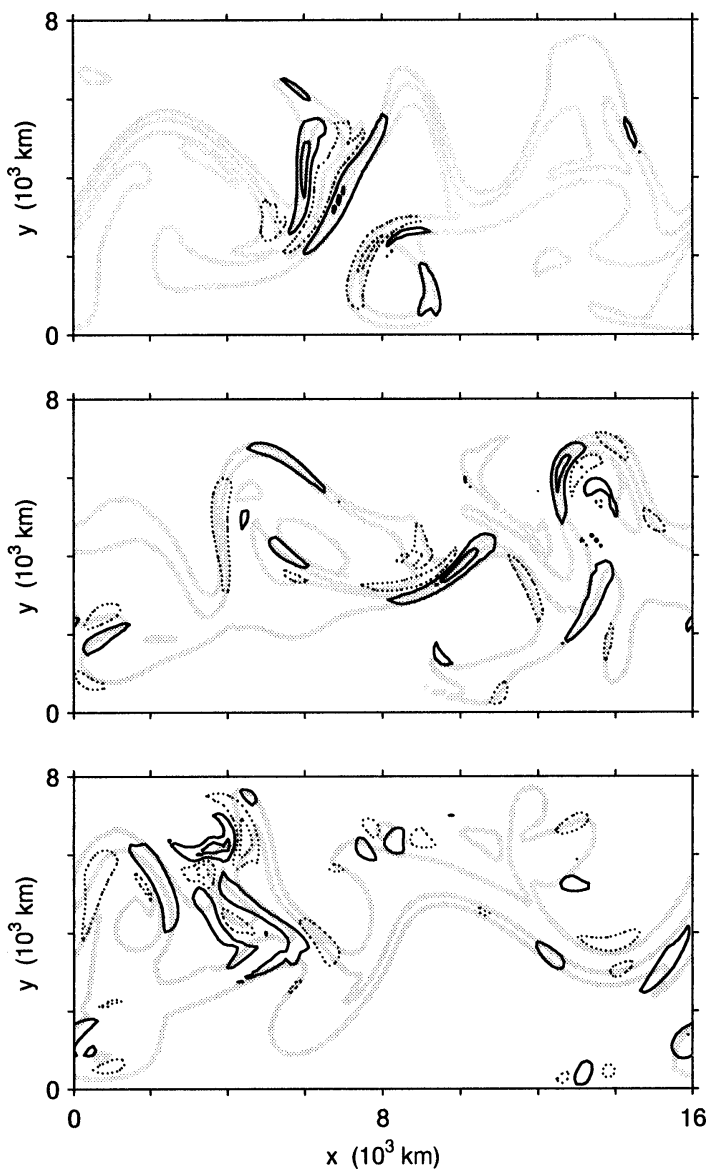

FIG. 4. Contours are shown at $1 / 4$ and $3 / 4$ of the (arbitrary) maximum amplitude (solid lines; negative values dotted). Gray lines are PV contours at $z / H=1$ for the unperturbed solution; the contour interval corresponds to about $20 \mathrm{~K}$ of potential temperature on the tropopause. The upper, middle, and lower panels show $t=120,140$, and 160 days, respectively.

turbations that grow as displacements or modifications of existing finite-amplitude features in an unperturbed flow.

The structure of the LVs in terms of other fields follows from the characteristics of the PV perturbations through the inversion of PV for streamfunction. The streamfunction perturbations are thus smoothed versions of the PV perturbations - they tend to be large where the unperturbed PV gradients are large, they have scales

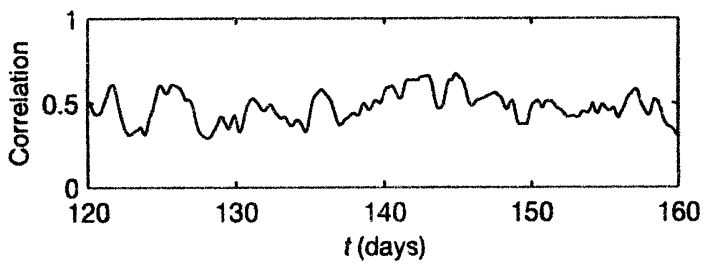

FIG. 5. The correlation, as a function of time, of the square of the $\mathrm{PV}$ at $z / H=1$ for the leading Lyapunov vector with the magnitude of the horizontal gradient of the unperturbed PV at the same level. 


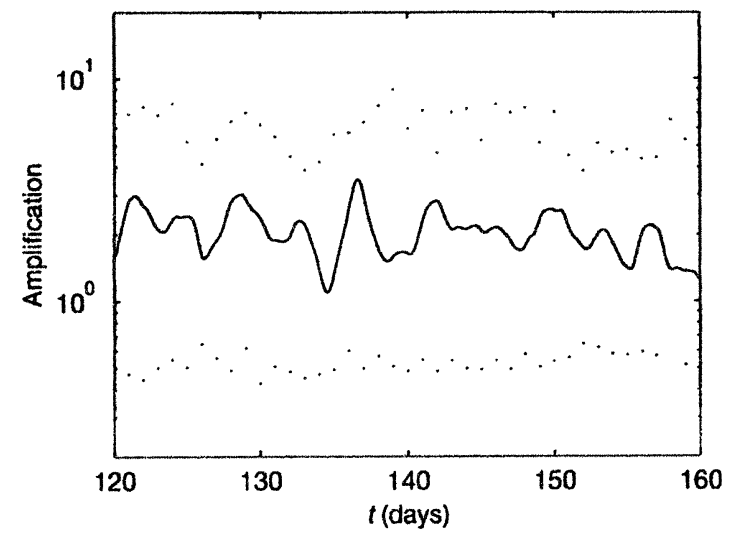

FIG. 6. Two-day amplifications for the leading Lyapunov vector (solid line) and for the first and last singular vectors (dots) constrained to the subspace spanned by the leading 20 Lyapunov vectors. Amplifications are for the (square root of) total energy.

comparable to those of the unperturbed streamfunction, and they often represent modifications or displacements of existing features. In the vertical, the streamfunction for $\mathbf{w}_{1}$ inherits the smooth interior structure and (typically) weak westward tilt with height of the baroclinic waves in the unperturbed solution.

Finally, all of the above characteristics are shared at least qualitatively by LVs beyond $\mathbf{w}_{1}$. The time-averaged correlation of the perturbation PV with the unperturbed PV gradient, for example, is between 0.5 and 0.6 for all of the first $60 \mathrm{LVs}$. The only systematic trend is that the first few LVs tend to be more spatially localized.

\section{d. Singular vectors confined to a Lyapunov subspace}

It is well known that, over a given finite time interval, there will be perturbations that amplify at a rate greater than $\lambda_{1}$. For example, as illustrated by Fig. 6, the local (in time) amplification of $\mathbf{w}_{1}$ may be substantially larger or smaller than that implied by $\lambda_{1}$. In addition, LVs other than $\mathbf{w}_{1}$ may have the largest amplification over a given time interval [see Fig. 4 of Vannitsem and Nicolis (1997)].

It is straightforward to calculate the perturbation within a given subspace that amplifies most under linearized dynamics over a given time interval. ${ }^{2}$ This perturbation is the leading singular vector for the propagator $\mathbf{M}$ constrained to the chosen initial subspace, and more generally, the calculation yields a set of singular

\footnotetext{
${ }^{2}$ Let $\mathbf{W}(\tau)$ be the matrix whose $i$ th column is $\mathbf{w}_{\mathrm{i}}(\tau)$ for the $N \mathrm{LVs}$ (or indeed let those columns be any set of orthogonal unit vectors spanning a subspace of interest). Next, define $\mathbf{X}(t)=\mathbf{M}(\tau, t) \mathbf{W}(\tau)$ and perform a singular value decomposition on $\mathbf{X}$, so that $\mathbf{X}=\mathbf{U} \mathbf{\Sigma} \mathbf{V}^{\mathbf{T}}$ where $\Sigma=\operatorname{diag}\left(\sigma_{1}, \ldots, \sigma_{N}\right)$ and $\mathbf{U}$ and $\mathbf{V}$ are orthogonal matrices. The maximum amplification is then $\sigma_{1}^{1 / 2}$ and the perturbation that achieves this is $\mathbf{W} \mathbf{v}_{1}$, where $\mathbf{v}_{1}$ is the first column of $\mathbf{V}$. In general, the columns of WV are the initial singular vectors for the interval [ $\tau$ $t$ ] constrained to the column space of $\mathbf{W}$.
}

vectors that span the chosen initial subspace. Swanson et al. (2000) present a similar calculation in the context of a different quasigeostrophic model.

The maximum and minimum amplification factors for the subspace spanned by the first 20 LVs are also shown in Fig. 6 for a sequence of 2-day intervals. On average, the maximum amplification is 6.1 , as compared to the 2 -day amplification of about 2 implied by $\lambda_{1}$. In addition, there is typically a perturbation that decays by about a factor of 2 over the same intervals, even though all the perturbations in the subspace grow asymptotically. For the subspaces spanned by the first 35 and first $60 \mathrm{LVs}$, the average maximum amplifications are 7.6 and 9.4, respectively, and the average minimum amplifications are 0.37 and 0.28 .

The structure of the perturbation with largest amplification is, not surprisingly, qualitatively similar to that of the leading LVs. In particular, its PV is strongly correlated with the unperturbed PV gradient and it possesses scales (at least along PV contours) comparable to the unperturbed solution. The perturbation with largest amplification also typically does not resemble any single LV, but has significant projection on several.

\section{Summary and discussion}

For the quasigeostrophic baroclinic jet considered here, the leading LVs inherit many characteristics of the unperturbed flow. In particular, like the unperturbed flow, the LVs have strongest PV perturbations at the upper and lower boundaries. Consistent with this PV structure, the wind and temperature perturbations are also maximized at the boundaries and decay into the interior.

A key finding is that the PV perturbations associated with the leading LVs are strongly correlated with the unperturbed PV gradient. Qualitatively, these PV perturbations take the form of long strips, with scales comparable to those of the unperturbed flow, lying along contours of unperturbed PV in regions of large PV gradients. Adding such perturbations to the unperturbed solution displaces the PV contours coherently on the length scales of features in the unperturbed flow. Thus, the LVs represent local displacements or modifications of jets, waves and vortices that exist in the unperturbed solution. Snyder et al. (2003) discuss in more detail the tendency for perturbations to correlate with the unperturbed PV gradient.

To further illustrate the form of the LVs, consider the extreme example of a collection of point vortices in twodimensional nondivergent flow. This system is chaotic and small changes to the initial conditions will grow (on average) exponentially. Regardless of the form of the initial perturbation, however, a perturbed solution will eventually differ from an unperturbed solution only in the locations of the vortices. [Boffetta et al. (1997) show decaying two-dimensional turbulence behaves analogously, in that small perturbations predominantly 
produce displacements of the coherent vortices.] The LVs for the baroclinic jet considered here are similar, but may change the amplitude or shape, as well as the location, of coherent structures in the flow.

A natural question is the relation between properties of our discretized system and those of the continuous partial differential equations (PDEs) for quasigeostrophic flow. There is no general theory available that describes the correspondence between LVs and Lyapunov exponents for a PDE and those for a discrete system approximating the PDE. In the present quasigeostrophic model, we expect that the leading Lyapunov exponent and the dimension of the unstable subspace will increase indefinitely in the limit of increasing model resolution and decreasing model dissipation, and that the leading LVs will decrease in scale. Heuristically, this is because smaller spatial scales have faster timescales, at least until the dissipation scale is reached (Hoyer and Sadourny 1982, their section 5). Thus, our results are likely to apply directly only to sufficiently coarse-grained or dissipative quasigeostrophic solutions. In the real atmosphere, of course, there are numerous processes, such as moist convection, that will also likely cause rapid divergence of solutions on subsynoptic scales, but which are not included in quasigeostrophic dynamics. Characterizing the LVs for higher-resolution and more realistic atmospheric flows remains for future work.

One point that we have touched upon only tangentially is the mechanism by which the LVs grow. As noted in section $4 \mathrm{c}$, the simplest analogy with instabilities on parallel flow seems inappropriate. Another possibility is the notion of Farrell and Ioannou (1999) that the growth of the LVs is a consequence of the time dependence of the unperturbed flow. While there is nothing in our results to contradict this, it is not obvious how to extend to the present case their examples, which simply make the basic-state time dependent in the parallel flow problem. A final possibility is the view put forward by Snyder and Joly (1998) and Snyder (1999), in which attention is focussed on flows with finite-amplitude structures and the potential for perturbation growth via displacements or modifications of those existing features. This view fits with the phenomenology of the LVs (see also Boffetta et al. 1997), but at present falls short of a comprehensive theory for the LVs.
Acknowledgments. Isla Gilmour provided a helpful review of an earlier version of the manuscript. This work began while TH was a postdoctoral fellow in the Advanced Study Program at NCAR. Both authors were also supported at NCAR in part by the U.S. Weather Research Program.

\section{REFERENCES}

Boffetta, G., A. Celani, A. Crisanti, and A. Vulpiani, 1997: Predictability in two-dimensional decaying turbulence. Phys. Fluids, 9, $724-734$.

Bretherton, F. P., 1966: Critical layer instability in baroclinic flows. Quart. J. Roy. Meteor. Soc., 92, 325-334.

Cross, M. C., and P. C. Hohenberg, 1993: Pattern formation outside of equilibrium. Rev. Mod. Phys., 65, 851-1112.

Davis, C. A., 1992: A potential vorticity diagnosis of the importance of initial structure and condensational heating in observed extratropical cyclogenesis. Mon. Wea. Rev., 120, 2409-2428.

Farrell, B. F., and P. J. Ioannou, 1999: Perturbation growth and structure in time-dependent flows. J. Atmos. Sci., 56, 3622-3639.

Hamill, T. M., C. Snyder, and R. E. Morss, 2002: Analysis-error statistics of a quasigeostrophic model using three-dimensional variational assimilation. Mon. Wea. Rev., 130, 2777-2790.

Hoskins, B. J., M. E. McIntyre, and A. W. Robertson, 1985: On the use and significance of insentropic potential vorticity maps. Quart. J. Roy. Meteor. Soc., 111, 877-946.

Hoyer, J.-M., and R. Sadourny, 1982: Closure modeling of fully developed baroclinic instability. J. Atmos. Sci., 39, 707-721.

Legras, B., and R. Vautard, 1995: A guide to Lyapunov vectors. Proc. ECMWF Seminar on Predictability, Vol. 1, Reading, United Kingdom, ECMWF, 143-156.

Métais, O., and M. Lesieur, 1986: Statistical predictability of decaying turbulence. J. Atmos. Sci., 43, 857-870.

Reynolds, C. A., and R. M. Errico, 1999: Convergence of singular vectors toward Lyapunov vectors. Mon. Wea. Rev., 127, 23092323.

Snyder, C., 1999: Error growth in flows with finite-amplitude waves or other coherent structures. J. Atmos. Sci., 56, 500-506.

_- and A. Joly, 1998: Development of perturbations within a growing baroclinic wave. Quart. J. Roy. Meteor. Soc., 124, 19611983.

— covariances in a quasigeostrophic model. Mon. Wea. Rev., 131, 189-205.

Swanson, K. L., R. Vautard, and C. Pires, 1998: Four-dimensional variational assimilation and predictability in a quasi-geostrophic model. Tellus, 50A, 369-390.

— T. N. Palmer, and R. Vautard, 2000: Observational error structures and the value of advanced assimilation techniques. $J$. At mos. Sci., 57, 1327-1340.

Toth, Z., and E. Kalnay, 1997: Ensemble forecasting at NCEP and the breeding method. Mon. Wea. Rev., 125, 3297-3319.

Vannitsem, S., and C. Nicolis, 1997: Lyapunov vectors and error growth patterns in a T21L3 quasigeostrophic model. J. Atmos. Sci., 54, 347-361. 